\title{
A Rapid and Reliable Method for the Clonal Isolation of Acanthamoeba from Environmental Samples
}

\author{
Janice Zanella ${ }^{1,2}$, Sergio Olavo Pinto da Costa $^{3}$, Jucimar Zacaria ${ }^{1}$ and Sergio \\ Echeverrigaray $^{1 *}$ \\ ${ }^{1}$ Instituto de Biotecnologia; Universidade de Caxias do Sul; Caxias do Sul - RS - Brasil. ${ }^{2}$ Departamento de \\ Citopatologia e Microbiologia; Faculdade de Biomedicina; Universidade de Cruz Alta; RS - Brasil. ${ }^{3}$ Universidade \\ Católica de Santos; São Paulo - SP - Brasil
}

\begin{abstract}
Acanthamoeba are abundant in a wide range of environments, and some species are responsible for cutaneous infections, keratitis, and granulomatous amoebic encephalitis (GAE). The conventional detection and isolation of amoeba from clinical and environmental samples involves sampling and culture on non-nutrient Ágar medium. Although efficient, this system requires several transfers in order to eliminate contaminants, and is not appropriate for the isolation of individual amoeba from samples with a biodiverse community. In this study we propose an alternative method for the isolation of monocystic clones of Acanthamoeba. The propose method involves sampling, enrichment, encystment induction, and direct cysts micromanipulation and culture on Ágar plates.
\end{abstract}

Key words: Acanthamoeba, monocystic culture, micromanipulation

\section{INTRODUCTION}

Free-living amoebae are abundant in soil, dust and water. These organisms have been isolated from fresh water, seawater, surface water, swimming pools, mineral water, dust, contact lens solutions, among other environments (Khan 2006; Visvesvara et al. 2007; Caumo et al. 2009; Costa et al. 2010). Several amphizoic amoebae such as Acanthamoeba spp., Balamuthia spp. and Naegleria spp. occasionally invade hosts and cause infections (Khan 2003; Schuster and Visvesvara 2004; Khan 2006). Acanthamoeba can invade the human's skin, eye and central nervous system where they can be responsible for cutaneous infections, keratitis, and granulomatous amoebic encephalitis (GAE), respectively (Khan, 2003, 2006). In Brazil, a twenty year survey of
Acanthamoeba keratitis showed that amoebic corneal infection could be considered a wellestablished and increasing ophthalmological disease (Carvalho et al. 2009). Moreover, Acanthamoeba serves as a carrier for different microorganisms to humans, acting as a vehicle for the circulation of pathogens between the environment and humans (Krusnell and Linder, 2001; Huws et al. 2008).

Various Acanthamoeba species have been reported to be able to cause keratitis and other human infections, among which the most prevalent are $A$. castellani, A. polyphaga, A. rhysodes and $A$. hatchetti (Walochnik et al. 2000a; Khan 2006). Although easily recognized at the genus level by their polygonal cysts, the accurate Acanthamoeba species determination and pathogenic potential involves molecular analysis (PCR, RFLP-PCR or

*Author for correspondence: selaguna@ucs.br 
DNA sequencing) and several behavioral tests (osmotolerance, temperature tolerance and cytotoxicity assays) which can require axenic and clonal amoeba cultures (Walochnik et al. 2000b). Agar culture is the mainstay for laboratory detection of Acanthamoeba from clinical and environmental samples. Amoeba isolation and culture procedures involves sampling, plating on non-nutrient agar medium covered by Escherichia coli, incubation for 14 days at $30-37^{\circ} \mathrm{C}$ with periodical examination, amoeba harvesting at a non-contaminated site of the plate, and transfer to a fresh plate in order to eliminate ciliates, flagellates, bacteria and fungi. The overall isolation of Acanthamoeba is time consuming ( 7 to 60 days) and several samples, particularly environmental samples, are often lost due to fungal or bacterial contamination.

In order to solve these problems, efforts were made in this work to develop a rapid and reliable protocol for the clonal isolation of Acanthamoeba from environmental samples.

\section{MATERIAL AND METHODS}

\section{Acanthamoeba strains and sampling}

An Acanthamoeba polyphaga strain isolated from a non-hospital environment in Santos, SP, Brazil (Teixeira et al. 2009) was used in initial experiments to optimize the culture and encystment conditions. Ten samples were collected in community bathrooms of a public hospital in Caxias do Sul, RS, Brazil. Fresh water samples $(200 \mathrm{ml})$ were collected directly from the taps, and dust samples were collected by scrapping the surface $\left(100 \mathrm{~cm}^{2}\right)$ of basin benches using sterile swabs. All the samples were processed within $2 \mathrm{~h}$.

\section{Optimization of procedures for the clonal isolation of Acanthamoeba}

In order to evaluate the best condition for the enrichment and encystment of Acanthamoeba samples, three culture media were tested: (1) NNEI, non-nutritive medium (Page saline solution$2.5 \mathrm{mM} \mathrm{NaCl}, 1 \mathrm{mM} \mathrm{KH} \mathrm{PO}_{4}, 0.5 \mathrm{mM} \mathrm{Na}_{2} \mathrm{HPO}_{4}$, $40 \mathrm{mM} \mathrm{CaCl}_{2}$, and $20 \mathrm{mM} \mathrm{MgSO}_{4}$ ) seeded with 0.1 $\mathrm{ml}$ of a thermal inactivated 48-h culture of $E$. coli ATCC 11775, (2) NN-E, non-nutritive medium seeded with $0.1 \mathrm{ml}$ of an active 48-h culture of $E$. coli, and (3) PYG medium [proteose peptone $0.75 \%(w / v)$, yeast extract, $0.75 \%(w / v)$ and glucose $1.5 \%(w / v)]$. Acanthamoeba growth was monitored by direct microscopic counting of trophozoites and cysts using a Neubauer hemocytometer.

Otherwise specified, Acanthamoeba were cultured at $30-32^{\circ} \mathrm{C}$, and encystment was induced by incubation at low temperature $\left(4^{\circ} \mathrm{C}\right)$. Cyst isolation was performed using an Eclipse E 50i microscope with a TDM 50i yeast tetrads micromanipulator (Schuett-Biotec, Denmark) that allowed direct cyst isolation on the solid media. The three enrichment media described before were solidified with $1.0 \%$ agar (NNA-EI, NNA-E, and PYGA) and tested for micromanipulation. In order to compare the efficiency of the proposed method, the samples were also processed by the conventional plating method adopted by several authors (Caumo et al. 2009; Teixeira et al. 2009; Costa et al. 2010), and preconized by the UK National Health Service (www.hpa-standardmethods.org.uk).

\section{Processing and culture of environmental samples}

Fresh water samples $(50 \mathrm{ml})$ were centrifuged in Falcon tubes $(1000 \mathrm{xg}, 5 \mathrm{~min})$ and the pellet was suspended in $1.0 \mathrm{ml}$ NN-EI broth. Dust samples were directly suspended in $1.0 \mathrm{ml} \mathrm{NN}$-EI broth. For the conventional method, these samples were immediately plated on pre-seeded NNA-EI, sealed with plastic film, and incubated at $30^{\circ} \mathrm{C}$. For the proposed method, the samples were enriched by incubation for $48 \mathrm{~h}$ at $30^{\circ} \mathrm{C}$, transferred to $4^{\circ} \mathrm{C}$ for $24 \mathrm{~h}$ to induce cyst formation, and microscopically monitored. Encysted samples were centrifuged (1000 x g, $5 \mathrm{~min}$ ) and suspended in 50-100 $\mu \mathrm{l}$ of Page's saline. For micromanipulation, a loop (approx. $5 \mu \mathrm{l}$ ) of the suspension was spread in a straight line along the edge of a well dried NNAEI plate. Under the microscope, individual cyst were picked up with a micromanipulator needle and transferred to new positions on the plate. After manipulation, the plates were sealed with plastic film and incubated at $30^{\circ} \mathrm{C}$. Plates were daily examined with an inverted microscope, and actively growing amoebas from individual clones were transferred to fresh NNA-EI medium. Up to ten individual clones of positive samples were isolated and evaluated.

\section{Morphological and molecular characterization of Acanthamoeba isolates}

Amoeba cysts were recovered from the cultures, stained with haematoxylin/eosin (Grossniklaus et 
al. 2003), directly observed under the microscope at a 400X magnification, and identified as belonging to one of the cyst morphological groups (Acanthamoeba sp. I to III) as established by Pussard and Pons (1977). Molecular characterization of Acanthamoeba cultures was performed by RFLP-PCR of ssu rDNA according to Kong and Chung (1996).

\section{RESULTS}

Optimization of Acanthamoeba isolation method

Initial experiment of enrichment and cyst induction performed with an environmental isolate of A. polyphaga indicated that NN-EI broth supported better growth of trophozoites than NN-E and PYG media. Static culture of $A$. polyphaga trophozoites for 24, 48 and $72 \mathrm{~h}$ in NN-EI broth allowed 2x, 5x and 6x increase of $A$. polyphaga, respectively. The transfer of active enrichment cultures to low temperature $\left(4^{\circ} \mathrm{C}\right)$ for a period of $48 \mathrm{~h}$ induced up to $80 \%$ encystment. This percentage was not significantly increased by longer incubation (48h).

For micromanipulation, NNA-EI allowed a rapid and efficient isolation of Acanthamoeba cysts, as well as their hatching and trophozoites multiplication. Conversely, the presence of active E. coli on NNA-E posse several problems for the separation of cysts by drastic modification on surface tension that made it difficult for the attachment of the cyst to the needle. PYG did not support a good growth of Acanthamoeba increasing from $96 \mathrm{~h}$ to more than $168 \mathrm{~h}$ the growing period necessary for the first sub-culture of the clones.

\section{Application of the proposed method to environmental samples}

After established the above basic conditions, ten samples (five fresh water and five dust samples) were processed by the conventional plating method and the proposed method summarized in Figure 1. Five of the ten environmental samples examined using the proposed method (Fig. 1) were positive for Acanthamoeba (Table 1). Overall cyst viability was $82 \%$ (37 growing clones from 45 isolated cysts). Two samples (W1 and D2) exhibited just one Acanthamoeba species, while in three samples (W3, D1 and D5), two species were identified. The most prevalent species was $A$. castellanii identified in four out of five positive samples. This species was the only Acanthamoeba isolated from the samples $\mathrm{W} 1$ and D1, or was found associated with $A$. polyphaga (samples W3 and D1) and A. lenticulata (sample D5). $A$. castellanii was characterized by small cysts with stellate endocysts (Group II) and a typical RFLPPCR pattern (Fig. 2).

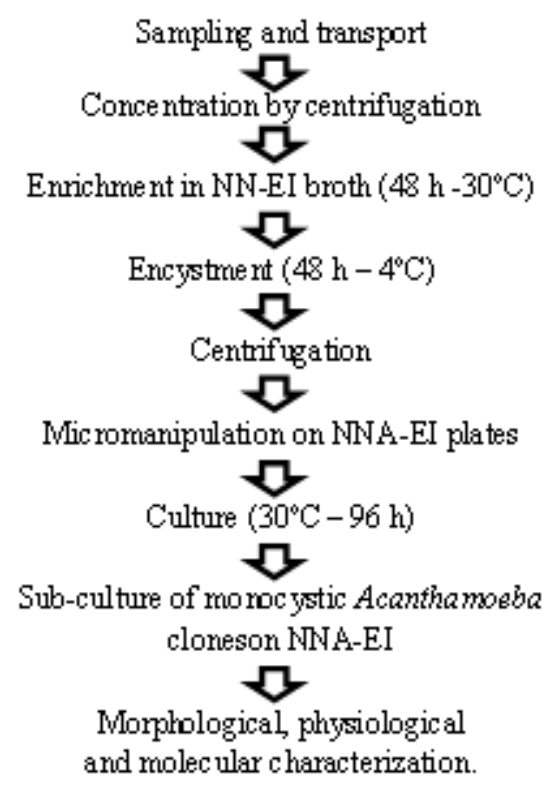

Figure 1 - Flowchart of the optimized process for the isolation of monocystic Acanthamoeba clones. 
Table 1- Samples and clonal isolates of Acanthamoeba obtained by micromanipulation method.

\begin{tabular}{cccccc}
\hline Sample & Origin & $\mathbf{N}^{\mathbf{0}}$ clones & $\begin{array}{c}\text { Clone } \\
\text { Name }\end{array}$ & $\begin{array}{c}\text { Cyst } \\
\text { Type }^{2}\end{array}$ & $\begin{array}{c}\text { Molecular } \\
\text { Classification }^{3}\end{array}$ \\
\hline W1 & Water & $\mathbf{5}$ & W1.1 & II & A. castellanii \\
W2 & Water & $\mathbf{0}$ & - & - & - \\
W3 & Water & $\mathbf{4}$ & W3.1 & II & A. castellanii \\
& & $\mathbf{2}$ & W3.2 & & A. polyphaga \\
W4 & Water & $\mathbf{0}$ & - & - & - \\
W5 & Water & $\mathbf{0}$ & - & - & - \\
D1 & Dust & $\mathbf{2}$ & D1.1 & II & A. castellanii \\
& & $\mathbf{6}$ & D1.2 & II & A. polyphaga \\
D2 & Dust & $\mathbf{1 0}$ & D2.1 & II & A. castellanii \\
D3 & Dust & $\mathbf{0}$ & - & - & - \\
D4 & Dust & $\mathbf{0}$ & - & - & - \\
D5 & Dust & $\mathbf{7}$ & D5.1 & II & A. castellanii \\
& & $\mathbf{1}$ & D5.2 & III & A. lenticulata \\
\hline
\end{tabular}

\footnotetext{
${ }^{1}$ Samples with the same number were collected from the same bathroom (ex. W1 and D1).

${ }^{2}$ Based on cyst morphology (Pussard and Pons, 1977).

${ }^{3}$ Based on the restriction patterns (MboI, HaeIII and TaqI) of the ssu rRNA amplicon (Kong and Chung, 1996).
}

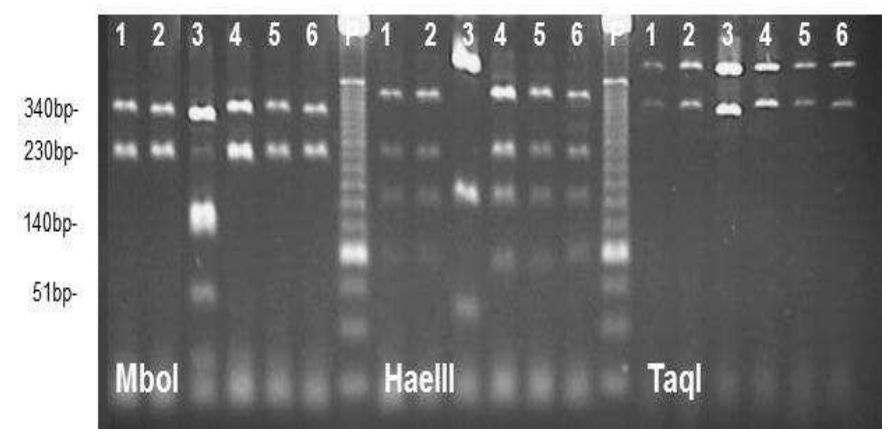

Figure 2 - ssu rDNA RFLP-PCR profiles of Acanthamoeba clones- A. castellanii (1- W1.1, 2W3.1, 4- D1.1, 5- D2.1, 6- D5.1) and A. polyphaga (3- W3.2). P- Molecular marker 100bp ladder.

A. polyphaga was identified in three samples: W3 and D1 associated with $A$. castellanii, and D2, in which it was the only Acanthamoeba species identified (Table 1). A. lenticulata was present in the sample D5 associated with A. castellanii. Using the conventional plating method, sample W1 contaminated with fungi preventing Acanthamoeba isolation, only A. castellani was isolated from sample D5, and at least three subcultures were necessary to decontaminate the positive samples.

\section{DISCUSSION}

Despite the lack of information about the efficiency of Acanthamoeba isolation methods, most of the works report difficulties with fungal, flagellate protozoa and bacterial contamination that demand several sub-cultures in order to obtain pure cultures (Walochnik et al. 2000b; Caumo et al. 2009; Teixeira et al. 2009; Costa et al. 2010). In the proposed method, these difficulties were overcome by the direct micromanipulation of induced cysts on NNA-EI plates. Although Acanthamoeba micromanipulation has been reported in other works (Fritsche et al. 1993; Kong and Chung 1996; Petry et al. 2006), this is the first report that used cyst induction and a yeast tetrad micromanipulator for the isolation and culture of individual cyst clones in a single step.

A comparison of the results obtained by the conventional plating and the current method indicated that the proposed protocol was more 
efficient and rapid than the plating method. The proposed method allowed isolating Acanthamoeba clones from all the positive environmental samples in 10 to 12 days, whereas the conventional plating method required 27 to 32 days to obtain axenic cultures. Even after several transfers, it was not possible to clean up one of the samples processed by the conventional method.

The main objective of this work was not to determine the incidence of Acanthamoeba, but to evaluate the efficiency of the proposed method. Nevertheless as observed in Table 1, five out of ten samples $(50 \%)$ collected at community bathrooms of a public hospital (two fresh water and three dust samples) exhibited the presence of Acanthamoeba. Compared with other Brazilian studies, the prevalence of Acanthamoeba was lower than that obtained by the analysis of dust samples from a university hospital in Curitiba, PR (Costa et al. 2010), but higher than that reported in the hospitals of Porto Alegre, RS (Carlesso et al. 2007, 2010) and Santos, SP (Teixeira et al. 2009). Considering the ubiquitous nature of Acanthamoeba, and the large number of factors that influenced their presence in a hospital environment, it was not surprising to obtain high but variable incidence of these protozoa.

Three out of five positive samples (60\%) exhibited the presence of two Acanthamoeba species. The simultaneous presence of Acanthamoeba species has been reported in clinical as well as in environmental samples (Khan 2006). Cystmorphology and RFLP-PCR analysis made it possible to detect three species of Acanthamoeba: A. castellanii, A. polyphaga and A. lenticulata. A. castellanii was the most prevalent and present in all the positive samples.

The present results showed that $50 \%$ of the samples (fresh water and dust) collected from a hospital environment in the Southern Brazil harbored potentially pathogenic species of Acanthamoeba (A. castellanii and A. polyphaga), representing a risk for patients and visitors. These data corroborated those of previous works developed in different Brazilian hospitals (Carlesso et al. 2007; Texeira et al. 2009; Carlesso et al. 2010; Costa et al. 2010), and emphasized the need for increasing the public awareness and hospital disinfection measures in order to reduce the incidence of Acanthamoeba infections (Carvalho et al. 2009).

\section{REFERENCES}

Carvalho FRS, Foronda AS, Mannis MJ, Höfling-Lima AL, Belfort RJr, Freitas D, Twenty years of Acanthamoeba keratitis. Cornea. 2009; 28: 516-519.

Carlesso AM, Simonetti AB, Artuso GL, Rott MB. Isolamento e identificação de amebas de vida livre potencialmente patogênicas em amostras de ambientes de hospital público da cidade de Porto Alegre, RS. Rev Soc Bras Med Trop. 2007; 40: 316320.

Carlesso AM, Artuso GL, Caumo K, Rott MB. Potentially pathogenic Acanthamoeba isolated from a hospital in Brazil. Curr. Microbiol. 2010; 60: 185190.

Caumo K, Frasson AP, Pens CJ, Panatieri LF, Frazzon AP, Rott MB. Potentially pathogenic Acanthamoeba in swimming pools: a survey in southern Brazilian city of Porto Alegre. Ann Trop Med Parasitol. 2009; 103: 477-485.

Costa AO, Castro EA, Ferreira GA, Furst C, Crozeta MA, Thomaz-Soccol V. Characterization of Acanthamoeba isolates from dust of a public hospital in Curitiba, Paraná, Brazil. J Eukar Microbiol. 2010; 57: $70-75$

Fritsche TR, Gautom RK, Seyedirachti S, Bergeron DL, Lindquist TD. Occurrence of bacterial endosymbionts in Acanthamoeba spp. Isolated from corneal and environmental specimens and contact lenses. J Clin Microbiol., 1993; 31: 1122-1126.

Grossniklaus HE, Waring GO, Akor C, CastellanoSanchez A, Bennett K. Evaluation of hematoxylin and eosin and special stains for the detection of Acanthamoeba keratitis in penetrating keratoplasties. Am J Ophthalmol. 2003; 136: 520-526.

Huws SA, Morley RJ, Jones MV, Brown MRW, Smith AW. Interactions of some common pathogenic bacteria with Acanthamoeba polyphaga. FEMS Microbiol Lett. 2008; 282: 258-265.

Khan NA. Pathogenesis of Acanthamoeba infections. Microb Pathog. 2003; 34: 277-328.

Khan NA. Acanthamoeba: biology and increasing importance in human health. FEMS Microbiol Rev. 2006; 30: 564-595.

Kong HE, Chung DI. PCR and RFLP variation of conserved region of small subunit ribosomal DNA among Acanthamoeba isolates assigned to either A. castellanii or A. polyphaga. Korean J Parasitology 1996; 34: 127-134.

Krusnell J, Linder E. Bacterial infections of free-living amoebae. Res Microbiol. 2001; 152: 613-619.

Petry F, Torzewski M, Bohl J, WilhelmSchwenkmezger T, Scheid P, Walochnik J, et al. Early diagnosis of Acanthamoeba infection during routine cytological examination of cerebrospinal fluid. J Clin Microbiol. 2006; 44: 1903-1904. 
Pussard M, Pons R. Morphologie de la paroi kystique et taxonomie du genre Acanthamoeba (Protozoa, Amoebida). Protistologica. 1977; 8: 557-598.

Schuster FL, Visvesvara GS. Free-living amoebae as opportunistic and non-opportunistic pathogens of humans and animals. Int J Parasitol. 2004; 34: 10011027.

Teixeira LH, Rocha S, Pinto RMF, Caseiro MM, Costa SOP. Prevalence of potentially pathogenic free-living amoebae from Acanthamoeba and Naegleria genera in non-hospital, public, internal environments from the city of Santos, Brazil. Braz J Infect Dis. 2009; 13: 395-397.

Visvesvara GS, Moura H, Schuster FL. Pathogenic and opportunistic free living amoebae: Acanthamoeba spp., Balamuthia mandrillaris, Naegleria fowleri, and Sappinia diploidea. FEMS Immunol Med Microbiol. 2007; 50: 1-26.
Walochnik J, Haller-Shober EM, Kölli H, Picher O, Obwaller A, Aspöck H. Discrimination between clinically relevant and nonrelevant Acanthamoeba strains isolated from contact lens-wearing keratitis patients in Austria. J Clin Microbiol. 2000a; 38: 3932-3936.

Walochnik J, Obwaller A, Aspöck H. Correlations between morphological, molecular biological, and physiological characteristics in clinical and nonclinical isolates of Acanthamoeba spp. Appl Environ Microbiol. 2000b; 66: 4408-4413.

Received: November 17, 2010; Revised: March 28, 2011; Accepted: September 27, 2011. 